\title{
Massive-scale RNA-Seq experiments in human genetic diseases
}

\author{
Valerio Costa', Marianna Aprile', Roberta Esposito', Maria Rosaria Ambrosio', Margherita \\ Scarpato', Carmela Ziviello', Italia De Feis ${ }^{2}$, Claudia Angelini ${ }^{2}$ and Alfredo Ciccodicola, \\ 'CNR, Institute of Genetics and Biophysics "A. Buzzati-Traverso" (IGB), Naples, Italy \\ ${ }^{2} \mathrm{CNR}$, Istituto per le Applicazioni del Calcolo (IAC), \\ Naples, Italy \\ http://www.igb.cnr.it
}

Since 2008, our research group is actively working in the field of NGS, with particular attention to RNA-Seq as innovative approach to understand cells' transcriptome in disease states (Costa et al., 2010a). In particular, combining molecular biology and computational expertise, we have recently analysed (Costa et al., 2011) by RNA-Seq - for the first time in Down syndrome (DS) - the global transcriptome of endothelial progenitor cells (EPCS), morphologically and functionally impaired in DS (Costa et al., 2010b). After rRNA depletion - followed by strand specific sequencing - we measured expression from (even) low expressed genes, we identified new regions of active transcription outside annotated loci, novel splice isoforms and extended untranslated regions for known genes, potentially new microRNA targets or regulatory sites. However, although RNA-Seq provided a huge amount of useful data for DS, showing a genome-wide alteration of gene expression (not limited to HSA21 genes), the experiment revealed only a fraction of the underlying complexity, giving no information about the reasons of such global deregulation. Therefore, in this ongoing project we aim to study: 1) by ChIP-Seq, the binding maps of some (preliminarily selected) transcription factors (TFs), key players in gene expression modulation, and 2) by RNA-Seq, the related gene expression changes in the same cells. ChIP-Seq, combining standard chromatin immunoprecipitation and massively parallel sequencing, allows to identify DNA sequences bound by TFs in vivo, helping to decipher gene regulatory networks (Park 2009). We believe that integrating RNA- and ChIP-Seq data would provide much more biological insights into gene expression regulation in DS cells, helping us to better understand some blood-related pathological aspects of the syndrome.

Our group is also participating to a large-scale collaborative industrial project aimed to develop a diagnostic kit for personalized therapeutic strategies in type 2 diabetic (T2D) patients resistant to conventional drug therapies. In particular, to elucidate some mechanisms of drug resistance, our group will perform massive-scale transcriptome analysis by RNA-Seq in a well-selected subset of individuals $(\sim 50)$, also collaborating with bioinformaticians to further data analysis.

In the light of these considerations, and given the objectives of the COST Action BM1006, our group will contribute to the goals of the SEQAHEAD project by actively integrating in the newborn European network of NGS, providing its expertise in sequencing technologies with a particular contribution (protocols, experimental data and pipelines for data analysis) to the RNA-Seq.

\section{References}

1. Costa V, Angelini C, De Feis I, Ciccodicola A. (2010) "Uncovering the complexity of transcriptomes with RNASeq." J Biomed Biotechnol. 2010:853916.

2. Costa V, Angelini C, D’Apice L, Mutarelli M, Casamassimi A, Sommese L, Gallo MA, Aprile M, Esposito R, Leone L, Donizetti A, Crispi S, Rienzo M, Sarubbi B, Calabrò R, Picardi M, Salvatore P, Infante T, De Berardinis P, Napoli C, Ciccodicola A. (2011) "Massive-scale RNA-Seq analysis of non ribosomal transcriptome in human trisomy 21." PLoS One. 6(4):e18493.

3. Costa V, Sommese L, Casamassimi A, Colicchio R, Angelini C, Marchesano V, Milone L, Farzati B, Giovane A, Fiorito C, Rienzo M, Picardi M, Avallone B, Marco Corsi M, Sarubbi B, Calabrò R, Salvatore P, Ciccodicola A, Napoli C. (2010) "Impairment of circulating endothelial progenitors in Down syndrome." BMC Med Genomics. 3:40.

4. Park PJ. ChIP-seq: advantages and challenges of a maturing technology. Nat Rev Genet. 2009 Oct;10(10):669-80. Epub 2009 Sep 8. 